take a line which is in conflict with the political views of the party in power. The Government should be open-minded in its approach to the issues raised by this Bill. There is no justification for its apparent wish directly to control the drug industry.

\section{Pulmonary Haemorrhage in the Newborn}

A number of necropsy studies have been done in an attempt to establish the factors that are responsible for deaths of newborn babies from haemorrhage into the lungs. ${ }^{1-4} \mathrm{~A}$ recent investigation by D. R. Shanklin and S. L. Wolfson seems to incriminate the use of oxygen as a possible cause of this condition. $^{5}$ It is very difficult to assess the true incidence of pulmonary haemorrhage in the newborn, as it occurs in two main forms. The first is associated with anoxia around the time of delivery. The haemorrhage is mainly subpleural and interstitial in distribution and there is, as a rule, less marked bleeding into the air spaces. The infants so affected are usually stillborn or die soon after delivery. The second form is seen in the infant who survives the immediate hazards of delivery only to die within a few days. The haemorrhage in this group of infants is predominantly intra-alveolar and the interstitial tissue may be unchanged or merely oedematous. The haemorrhage is commonly classified as "massive" or "severe." This type of haemorrhage is apparently not associated with any inherent bleeding diathesis, but it occurs as a terminal event in such a wide variety of conditions that it is difficult to assess it as an entity, and it is perhaps better regarded as a mode of death. Conditions which are known to terminate with pulmonary haemorrhage in the neonatal period include kernicterus, cerebral oedema, subdural haemorrhage, and pneumonia. Further confusion arises from the fact that some workers regard pulmonary haemorrhage as a component, together with pulmonary oedema and hyaline membrane formation, of the respiratory distress syndrome. ${ }^{67}$ Recently, however, A. J. McAdams ${ }^{4}$ was unable to confirm any common pathogenesis for pulmonary haemorrhage and hyaline membrane disease. There were two reasons for this lack of correlation. Firstly, the infants with pulmonary haemorrhage were not mainly premature-as is the case with infants suffering from the respiratory distress syndromeand secondly the age of death is greater in the former. If known associated conditions such as kernicterus, other cerebral lesions, and infection are eliminated, together with those infants known to have hyaline membrane, the remaining infants with "idiopathic" pulmonary haemorrhage account for $7 \%$ of deaths in the first week, an incidence of 0.9 per 1,000 live births. $^{8}$

So the incidence of this condition is not negligible, and these infants appear to drown in their own blood. The haemorrhage may occur at any time after delivery, but it is rare within the first six hours of life and the majority of these infants die between the third and fourth day. Though the grosser intracranial lesions such as kernicterus and subdural haemorrhage, which have known associations with pulmonary haemorrhage, should be excluded from the "idiopathic" group, nevertheless cerebral damage as a result of anoxia or cerebral oedema may be predisposing factors. ${ }^{4}$

The interesting feature of the report by Shanklin and Wolfson is their suggestion that therapeutic oxygen is a possible cause of this form of pulmonary haemorrhage. A total of 83 infants were studied at necropsy. Only eight of these weighed over $2,500 \mathrm{~g}$. at birth. Twelve infants were treated in air only, 11 were given oxygen at least six hours after delivery, 21 were given oxygen soon after delivery, and 39 were given oxygen continuously from birth. No pulmonary haemorrhage was seen in the lungs of infants kept in air or in those given oxygen after the first six hours of life. Fourteen of the 21 infants given oxygen soon after birth and 35 of the 39 infants given oxygen continuously had haemorrhagic lungs. These authors suggest that oxygen therapy for the first two hours of life only or when administered after the sixth hour of life does not cause pulmonary haemorrhage. Between two and six hours after delivery some change appeared to occur which led to bleeding into the lung. During the same period an infant maintained in air seems to be protected from this change. Their results in respect of hyaline membrane formation are also of some interest. Fifty-seven $(68.7 \%)$ of the 83 infants had hyaline membrane in their lungs, but the incidence was $87.2 \%$ in those given oxygen continuously. These findings reinforce those of other recent studies. $^{910}$ W. H. Northway, R. C. Rosan, and D. Y. Porter $^{9}$ describe a condition of "bronchopulmonary dysplasia " occurring as a result of treatment of infants with hyaline membrane by a respirator. Humidified oxygen at a concentration of $80-100 \%$ was administered in a positive pressure respirator. This seemed to result in a prolongation of the resolution of hyaline membrane and provoked squamous metaplasia of the bronchiolar epithelium and interstitial oedema and fibrosis. This could result in the continuation of respiratory symptoms well beyond the neonatal period. The diffuse pulmonary change did, however, seem to be reversible. That pulmonary lesions associated with oxygen therapy and artificial ventilation are not confined to the newborn has been shown by $G$. Nash, J. B. Blennerhassett, and $\mathbf{H}$. Pontoppidan. ${ }^{10}$ The pathological changes seen in the lungs of their adult patients included oedema, thickening of the interlobular septa, and hyaline membrane formation. Moreover, these changes were related to the use of high concentrations of oxygen rather than just to the length of time the respirator was used.

The implications of these studies for the clinical management of patients are not yet entirely clear, and it must be noted that they are based mainly on necropsies. Changes seen in the lungs post mortem may or may not reflect accurately alterations in vascular permeability during life. Probably, however, the administration of high concentrations of oxygen over a prolonged period affects not only the retinal vessels, leading to retrolental fibroplasia, but also the pulmonary vessels.

It has been known for many years that it is possible to produce pulmonary lesions in mice by giving oxygen at a concentration of more than $40 \%$, and about half the mice die if the concentration is raised to $80 \% .{ }^{11}$ These changes in

I Ahvenainen, E. K., and Call, J. D., Amer. 7. Path., 1952, 28, 1

2 Ahvenainen, E. K., Ann. paediat. Fenn., 1956, 2, 44.

- Esterly, J. R., and Oppenheimer, E. H., F. Pediat., 1966, 69, 3.

- McAdams, A. J., Amer. f. Dis. Child., 1967, 113, 255.

- Shanklin, D. R., and Wolfson, S. L., Nero Engl. F. Med., 1967, 277,

- Bound, J. P., Butler, N. R., and Spector, W. G., Brit. med. F., 1956, 2, 1191 .

- Rowe, S., and Avery, M. E., F. Pediat., 1966, 69, 12.

Rowe, S., and Avery, M. E., F. Pediat., 1966, 69, 12. Kellar, 1963, p. 203. London.

- Northway, W. H., Rosan, R. C., and Porter, D. Y., New Engl. \}. Med., 1967, 276, 357.

10 Nash, G., Blennerhassett, J. B., and Pontoppidan, H., New Engl. 3 Med., 1967, 276, 368

11 Lorrain Smith, J., f. Physiol. (Lond.), 1897, 22, 397.

12 New Engl. f. Med., 1967, 276, 412. 
the lungs include oedema, haemorrhage, and hyaline membrane formation. If, however, the affected animals are removed from the highly oxygenated environment and are returned to air they may recover completely. Oxygen therapy is thus a subject to be approached with some caution. ${ }^{12}$ A team of experts should be available to monitor the oxygen content of alveolar air and of the arterial blood in order that dangerously high concentrations may be avoided. Safe administration of oxygen also requires the judgement of the clinician on whether an infant may not be returned to air, at least for a period, without too great a risk of tissue anoxia.

\section{Coal Gas and the Brain}

The common methods of attempting suicide in Britain are inhalation of carbon monoxide and ingestion of barbiturates and aspirin. Accidental exposure to coal gas adds to the number of cases of carbon monoxide poisoning, so that each year it rises to several thousand. As the affinity of haemoglobin for carbon monoxide approaches 300 times that which it has for oxygen, and as nervous tissue is peculiarly and rapidly sensitive to oxygen lack, the major brunt of the toxic symptoms is borne by the central nervous system. In rapidly fatal cases of carbon monoxide poisoning the findings at necropsy are congestion and haemorrhages in all the organs, and these show the characteristic pink colour; but in less acutely fatal cases ischaemic changes, though seen, are less dramatic in other organs than are the anoxic and ischaemic lesions in the brain. Here there is necrosis in the second and third layers of the cortex and the superficial white matter, with degeneration of the Purkinje cells in the cerebellum and the cells of Sommer's sector of Ammon's horn. ${ }^{1}$ When death occurs later still there is extensive demyelination with gliosis and cerebral atrophy. Lesions may be found at all stages in the globus pallidus, and these are probably of vascular origin.

As the changes are widespread and diffuse, it is not surprising that the symptoms and sequelae of carbon monoxide intoxication cover almost the whole spectrum of cerebral dysfunction. What is surprising, however, is that patients who survive the acute stage only rarely suffer permanent effects. Death is common $(20-40 \%)$, but if the patient lives recovery is usually complete. In a survey in New York State of over 21,100 cases of carbon monoxide poisoning only 39 patients were found to have permanent sequelae. ${ }^{2}$

Early symptoms are also very varied. Euphoria, lack of judgement, headache, tightness across the forehead, and blurring of vision may all occur, progressing to confusion, disorientation, ataxia, drowsiness, and coma. Apart from the headache and visual symptoms the patient may be unaware of these features. If he is also unaware of the gas he may reach a state in which muscle anoxia prevents him from withdrawing from the danger even if consciousness is not lost early, as it will be if the patient is suddenly subjected to high concentrations. This is sometimes the fate of gallant would-be rescuers who have not paused to consider the possible cause of unconsciousness in a victim lying in an enclosed space.

Meyer, A., in Greenfield's Neuropathology, 2nd ed., 1963. London.

- Shillito, F. H., Drinker, C. K., and Shaughnessy, T. J., f. Amer. med. Ass., 1936, 106, 669.

- Smith, G., Ledingham, I. McA., Sharp, G. R., Norman, J. N., and Bates, E. H., Lancet, 1962, 1, 816.

- Garland, H., and Pearce, J., Quart. Ұ. Med., 1967, 36, 445

- Garland, H., and Prit. med. Ұ., 1965, $1,1232$.

- Plum, F., Posner, J. B., and Hain, R. F., Arch. intern. Med., 1962, 110,18 .
Treatment must be directed at rapid removal of the patient from the source of the gas, and then conversion of the carboxyhaemoglobin to oxyhaemoglobin. Pure oxygen or oxygen with $5-7 \%$ carbon dioxide should be given as quickly as possible, and in comatose patients the use of intravenous hypertonic solutions may serve to reduce cerebral oedema. However, where this is available, exposure to hyperbaric oxygen at $2-2 \frac{1}{2}$ atmospheres pressure is the treatment of choice, and in a series of 22 cases treated in this way in Glasgow a $100 \%$ recovery rate with no sequelae was obtained. ${ }^{3}$

The sequelae of carbon monoxide poisoning can vary from permanent confusion and disorientation to merely a generalized increase in tendon reflexes, or conversely signs of a peripheral neuropathy. All degrees of Parkinsonism and dystonia have also been recorded, though the frequency of this complication has probably been overemphasized. The physiology, pathology, and clinical manifestations of carbon monoxide poisoning as it affects the nervous system have been reviewed recently by the late Hugh Garland and John Pearce from Leeds." From a personal experience of an episode of acute poisoning affecting four young men almost simultaneously they have drawn attention not only to the very varied neurological picture-which in their cases included very marked signs of parietal lobe disorder, visual impairment of cortical origin, transient deafness, epilepsy, and mental changes ranging from retardation to frank psychosis-but also to the capacity for these signs to vary quite dramatically and for the patient to relapse after apparent recovery. One-third of the New York State cases showing sequelae showed recovery and relapse, ${ }^{2}$ and other cases are on record in which after periods ranging from a few days to several weeks relapse occurred, leading to akinesia, Parkinsonism, and death. ${ }^{56}$ F. Plum and his colleagues $^{6}$ believed this occurred in patients who were got out of bed too soon. Though relapse may be associated with the extensive demyelination, in practice recovery even from the relapse is usually complete.

The mortality rate for carbon monoxide poisoning is based on figures which often exclude many accidental cases of mild degree. Add to this the rarity of permanent ill effects, and it becomes clear that if immediate effective treatment is available-perhaps by means of flying-squad teams-and patients are not allowed to return to activity too soon the prognosis is generally good. It is a paradox that many patients might not wish it so.

\section{Treatment of Gonorrhoea}

In 1958 F. R. Curtis and A. E. Wilkinson ${ }^{2}$ reported that some strains of gonococci showed in-vitro evidence of becoming less sensitive to penicillin and that the reduction in sensitivity correlated with a higher clinical relapse rate. As a result of this and other similar reports most physicians increased the routine dose to at least 600,000 units of procaine penicillin or penicillin in aluminium monostearate (P.A.M). The latter had the disadvantage of a slow rise and fall in serum concentration, whereas Curtis and Wilkinson had pointed out that the ideal penicillin preparation for the cure of gonorrhoea should have a rapid rise, high plateau of 1.0 unit per $\mathrm{ml}$. serum for 18-24 hours, and then a rapid fall in concentration. At about the same time $P$. Durel ${ }^{2}$ had reported clinical and laboratory evidence of an increasing percentage of streptomycin-resistant gonococci.

Thus it was thought that reports of penicillin-resistant strains from individual clinics should be amplified by a wider 